\title{
Effects of Zingiber officinale hydro-alcoholic extract on HMG-COA reductase level in the testis of streptozotocin- induced diabetic rats
}

\author{
Bahman Moradi-Podeh $^{1}$, Alireza Kheirollah ${ }^{2}$, Fatemeh Ahmmadpour ${ }^{1}$, Nasrin Lamuchi-Deli ${ }^{1}$, Seyede-Arefe \\ Payami $^{1}$, Ghorban Mohammadzadeh ${ }^{3 *}$ \\ ${ }^{1}$ Department of Clinical Biochemistry, Faculty of Medicine, Ahvaz Jundishapur University of Medical Sciences, Ahvaz, Iran. \\ ${ }^{2}$ Cellular and molecular Research Center, Department of Clinical Biochemistry, Faculty of Medicine, Ahvaz Jundishapur University of Medical \\ Sciences, Ahvaz, Iran \\ ${ }^{3}$ Hyperlipidemia Research Center, Department of Clinical Biochemistry, Faculty of Medicine, Ahvaz Jundishapur University of Medical Sciences, \\ Ahvaz, Iran
}

\section{A R T I C L E I N F O}

Article Type:

Original Article

\section{Article History:}

Received: 20 October 2017

Accepted: 13 January 2018

\section{Keywords:}

Streptozotocin-induced diabetes HMG-COA reductase

Zingiber officinale Medicinal plant

\begin{abstract}
A B S T R A C T
Introduction: Zingiber officinale Roscoe, commonly known as ginger, is used as a cooking spice and therapeutically for its antioxidant and androgenic activities. We investigated the effects of $Z$. officinale hydro-alcoholic extract on HMG-CoA (3-hydroxy-3-methylglutaryl coenzyme A) reductase level in the testis of streptozotocin (STZ)-induced diabetic rats.

Methods: The current experimental study was performed on four groups of male Wistar rats one of them was kept as a healthy control, while the others were rendered diabetic via a single intraperitoneal injection of STZ $\left(60 \mathrm{mg} \mathrm{kg}^{-1}\right)$. One group was considered as diabetic control; while the others were given orally hydro-alcoholic extract (200 and $400 \mathrm{mg} \mathrm{kg}^{-1}$ ) for 56 consecutive days. Body weight, blood glucose and insulin concentrations were evaluated using standard methods. The HMG-COA reductase level was determined by western blot analysis. Results: Treatment with the extract resulted in a significant reduction of serum glucose concentration and HMG-COA reductase level in the rat's testis compared to diabetic controls $(P<0.01)$. A significant increase in body weight was observed in treated diabetic rats. Also, serum insulin was significantly increased in diabetic rats treated with $400 \mathrm{mg} / \mathrm{kg}$ of the extract compared to diabetic controls $(P<0.05)$.

Conclusion: Ginger has a potential influence on the regulation of cholesterol homeostasis by modulating of HMG-COA reductase level. The results provide scientific evidence to confirm the traditional use of $Z$. officinale in the treatment of diabetes mellitus.
\end{abstract}

Implication for health policy/practice/research/medical education:

Oral administration of ginger hydro-alcoholic extract could ameliorate hyperglycemic induced diabetic complications. Also, its potential influence in the regulation of cellular cholesterol homeostasis possibly is mediated by modulating of HMG-COA reductase level.

Please cite this paper as: Moradi-Podeh B, Kheirollah A, Ahmmadpour F, Lamuchi-Deli N, Payami SA, Mohammadzadeh G. Effects of Zingiber officinale hydro-alcoholic extract on HMG-COA reductase level in the testis of streptozotocin-induced diabetic rats. J Herbmed Pharmacol. 2018;7(2):94-99. doi: 10.15171/jhp.2018.16.

\section{Introduction}

Cholesterol is a vital constituent of mammalian cell membranes, and comprises about $30 \%$ of all animal cell membranes, it maintains membranes and modulates membrane fluidity over the range of physiological temperatures, however, excess cellular and circulating cholesterol is harmful that can be related to some diseases, including coronary artery disease and atherosclerosis $(1,2)$. The maintenance of cellular cholesterol homeostasis is precisely regulated by several feedback mechanisms. The major regulatory targets of these feedback mechanisms are HMG-CoA reductase in cholesterol biosynthesis pathway, the low-density lipoprotein (LDL) receptor in cholesterol uptake, and cholesterol 7a-hydroxylase in cholesterol 
catabolism pathway (3-5). Diabetes mellitus has been revealed to be associated with sexual dysfunction in both sexes.

Previous studies have shown that several phytoingredients have a potential influence in the regulation of cholesterol homeostasis in animal models (6-8). One of these phyto-ingredients is Zingiber officinal Roscoe, that is traditionally widely-utilized spice in worldwide. Some properties such as anti-inflammatory, antioxidant and anti-diabetic properties have been reported for Z. officinal (9-11). Some studies have shown that the ethanol extract of $Z$. officinale alleviates plasma lipids in cholesterol fed hyperlipidemia rabbits $(12,13)$ and in streptozotocin (STZ)-induced diabetic rats (14). Also, LDL oxidation in atherosclerotic mice was inhibited by ethanol extract of $Z$. officinale in some studies (15). In addition to the alcohol extract of ginger, the aqueous extract of $Z$. officinale has also been shown to reduce lipid profile in serum of normal rat (16). These reported effects of $Z$. officinale may be resulted from inhibition of cellular cholesterol biosynthesis (15) and excitation of cholesterol removal from the body $(17,18)$. To the best of our knowledge, the effects of hydro-alcoholic extract of $Z$. officinale on the HMG-CoA reductase level in the testis of STZ-induced diabetic rats, which is the main aim of this study, have not yet been well assessed.

\section{Materials and Methods}

Animals

In this experimental study a total 16 male Wistar rats with the average weight of 200-250 g were assessed. The rats were purchased from Research Center and Experimental Animal House of Ahvaz Jundishapur University of Medical Sciences (Ahvaz, Iran). Upon entrance, all the animals were accommodated in a well-ventilated room with a comparative temperature of $22 \pm 2^{\circ} \mathrm{C}$ and photoperiod of 12-hour light-dark cycle. During the experiment, all animals were given free access to standard food pellet and tap water.

\section{Experimental design}

For induction of diabetes, after one-week adaptation and following an overnight fasting, the rats were given a single intraperitoneal (ip) injection of STZ (Purchase from Sigma-Aldrich, USA) $60 \mathrm{mg} / \mathrm{kg}$ that was freshly dissolved in $0.1 \mathrm{M}$ cold citrate buffer in $\mathrm{pH}$ 4.5. Also, the healthy control rats were injected alone with the same volume of sodium citrate buffer solution. After three days of STZ induction, blood specimens were collected from the nicked tail-vein, and the blood glucose levels were measured. The blood glucose more than $350 \mathrm{mg} / \mathrm{dL}$ was considered as diabetes. Two weeks after STZ injection, oral gavage of $Z$. officinale extract was initiated and continued for a period of 8 weeks. The rats were randomly categorized into four experimental groups, each group consisted of four rats, and assessed as follows:
Group 1: Healthy control was orally gavaged with $1.5 \mathrm{~mL} /$ $\mathrm{kg}$ distilled water once daily.

Group 2: Diabetic control was orally gavaged with $1.5 \mathrm{~mL} /$ $\mathrm{kg}$ distilled water once daily.

Group 3: Diabetic rats were orally gavaged with $200 \mathrm{mg} / \mathrm{kg}$ of the extract once daily.

Group 4: Diabetic rats were orally gavaged with $400 \mathrm{mg} / \mathrm{kg}$ of the extract once daily.

\section{Sample collection}

The fasted rats in all groups at the end of eight-week treatment were weighed and then sacrificed after anesthetizing. By using not heparinized syringes, fresh blood samples were directly obtained via cardiac puncture and sera were separated by centrifugation for biochemical measurements. The testis was immediately isolated and washed with cold normal saline solution, then stored at $-80^{\circ} \mathrm{C}$ for later assays.

Preparation of Zingiber officinale hydro-alcoholic extract Dried roots of $Z$. officinale were purchased from a local company, Gol Darou, Isfahan, Iran. For extraction, 200 $\mathrm{g}$ of dry roots were grounded using an electric blender, and soaked in $1400 \mathrm{~mL}$ of $70 \%$ methanol solution (v/v) for 3 days. The extract was filtered through the Whatman filter paper, concentrated in a rotoevaporator, and dried with Freeze Dryer. The obtained extract, dry powder, was calculated $12.5 \%$.

\section{Western blot analysis}

Western blot Analysis was performed on the supernatant fraction of homogenized rat's testis. By Bradford method, concentrations of total protein on the supernatant were measured. Seventy micrograms of protein were loaded onto $8 \%$ sodium dodecyl sulfate (SDS)-polyacrylamide gel for electrophoresis (SDS-PAGE). After SDS-PAGE, by BIO-RAD transfer system (USA), Protein blots transferred to a methanol pre-activated polyvinylidene difluoride (PVDF) membrane. After blotting, the membrane was blocked with skimmed milk 5\% for 1.5 hours with gradually shakes. Subsequently, blots were probed with antibody specific for HMG-CoA reductase (1:5000 dilution) (Santa Cruz Biotechnology), and reprobed for $\beta$-actin (1:4000 dilution) (sc-130656; Santa Cruz Biotechnology) to serve as a loading control. Bound antibody was probed with secondary antibody, goat antirabbit IgG-HRP (horse radish peroxidase) (sc-2030; Santa Cruz) diluted 1:10000 in 3\% BSA (bovine serum albumin) for $1 \mathrm{~h}$ at room temperature. After each step membranes were washed thrice with Tris-buffered salineTween (TBST) $0.1 \%$. Finally, the blots were visualized with Chemi-Doc gel documentation system (Bio-Rad, Hercules, CA) using enhanced chemi luminescence (ECL) western blot detection kit according to the manufacturer's protocol. By using ImageJ software optical densities of bands were measured and quantified as ratio to $\beta$-actin. 
Statistical analysis

All statistical analysis was carried out with SPSS software (SPSS Inc., Chicago, IL). Statistical significance was determined by one-way analysis of variance (ANOVA) followed by Tukey post hoc test. The comparison of data with normal distribution including blood glucose and body weight was done by ANOVA test. And, the data with no normal distribution were analyzed by Kruskal-Wallis test. The results were reported as means \pm standard deviation (SD). A $P$ value $<0.05$ was considered statistically significant difference.

\section{Results}

Effects of Zingiber officinale extract on the biochemical parameters

Before induction of diabetes, the mean of body weight was similar between groups and there was no significant difference in fasting blood glucose concentration. The effect of $Z$. officinale extract on the body weight, insulin level, and fasting blood glucose concentrations were assessed and the results are presented elsewhere (19).

Effects of Zingiber officinale extract on the rats' testis HMG-COA reductase level

As illustrated in Figure 1, at the end of the experimental period in western blot analysis, a significant decrease in HMG-COA reductase protein level was observed in diabetic rats treated with 200 and $400 \mathrm{mg} / \mathrm{kg}$ of $Z$. officinale extract compared to controls $(P<0.01)$. No statistically significant difference for HMG-COA reductase protein level was observed between healthy controls and diabetic controls $(P=0.208)$.

\section{Discussion}

Hypercholesterolemia is common in diabetic patients and is a risk factor for coronary heart disease (CHD) according to reported by numerous studies (20-22). Some diabetic medications have troublesome side-effects, so there is increasing tendency to use of natural therapies. In traditional medicine, herbs are rich sources of therapeutic compounds for relieving symptoms of illness such as diabetes and hyperglycemia. Z. officinale is used as an important herbal remedy which has a reducing effect on blood glucose and cholesterol according to the results from previous studies $(9-11,23)$ However, no studies have been done about the effects of $Z$. officinale hydro-alcoholic extract on the HMG-COA reductase expression in testis of diabetic rats. In the present study we have investigated this issue.

Our results showed that in STZ-induced diabetic rats' body weight was significantly decreased compared to healthy control. Based on a study conducted by Kusari et al, in STZ-induced diabetes loss of body weight was observed. This reduction was related to loss of skeletal muscle resulting from hyperglycemia or high urine excretion and dehydration (24). Also, Eleazu et al showed that the reason for the loss of body weight was resulted from the degradation of muscle proteins similar to the results of our study (25). According to the present study, Z. officinale extract can prevent weight loss in rats with STZ-induced diabetic and this effect may be related to its hypoglycemic properties. Furthermore, this result is consistent with the

(A)
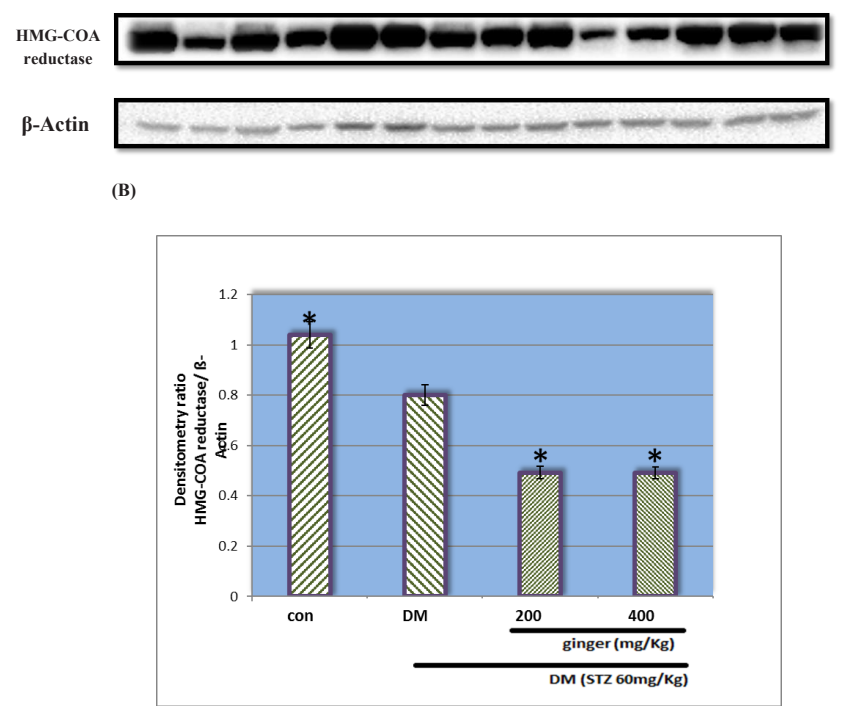

Figure 1 Effect of $Z$. officinal extract at the end of 8 weeks intervention on the HMG-COA reductase expression. (A) Representative of immune-blots demonstrating specific bands for HMG-COA reductase (B) Graphical presentation of data obtained from four independent experiments from western blot analysis. The mean value of HMG-COA reductase is expressed as ratio of HMG-COA reductase to $\beta$-actin in each column. $\beta$-actin was applied as an internal control. Error bars show S.D. ${ }^{*} P<0.01$ vs. healthy control and diabetic controls. con=Healthy control; DM=Diabetic control; 200= Diabetic treated with $200 \mathrm{mg} / \mathrm{kg}$ of the extract; 400=Diabetic treated with $400 \mathrm{mg} / \mathrm{kg}$ of the extract. In Figure 1A from left to right, lanes 1,2,3,4 related to con ; lanes 5,6,7,8 related to DM; lanes 9,10,11 related to 200; and lanes $12,13,14$ related to 400 , respectively. 
findings reported in previous studies $(26,27)$.

In the present study, serum insulin level was significantly reduced in diabetic control in comparison with healthy control, ones after 10 weeks of diabetes induction. It has been demonstrated the diabetogenic effect of STZ is resulted from the permanent destruction of the $\beta$-cells and deficiency of insulin secretion (28), so this finding can verify our result. On the other hand, serum insulin level was significantly higher in diabetic rats treated with $400 \mathrm{mg} / \mathrm{kg}$ of Z. officinale extract than diabetic controls $(29,30)$. Z . officinale extract may be stimulating $\beta$ cells to secrete insulin or releases this hormone of insulin-granule complex. This finding is in agreement with our results $(29,30)$. In diabetic controls compared to healthy control ones, following decreased insulin secretion, the fasting blood glucose concentration was significantly increased. Some other animal studies have reported similar findings $(11,28,29)$.

We observed that decrease of fasting blood glucose in diabetic rats treated with $Z$. officinale extract was dose dependent which is in agreement with other findings reported by other studies $(11,23,27)$. Hypoglycemic property of $Z$. officinal is probably related to certain plant components such as polyphenol constituents and flavonoids (9). Flavonoids may decrease plasma and hepatic cholesterol level due to the decrease hepatic HMG-CoA reductase level (31). Raw ginger extract can decrease serum cholesterol and triacylglycerol levels in diabetic rats and it has the great effect for controlling of diabetic complications (32). Our results indicated there was a significant reduction in HMG-CoA reductase level after treatment with both doses of $Z$. officinal extract. Cholesterol is a precursor to testicular androgens production in Leydig cells. It was assumed that decrease of total cholesterol level by reduction of HMG-CoA reductase with $Z$. officinal may create sexual hormone dysfunction and it is a side effect to use for diabetes disease. But Kamtchouing et al reported that the aqueous extract of $Z$. officinal significantly increases the serum testosterone level (33) that is in contrast with its cholesterol-lowering effect. The mechanism of action of ginger for increasing the level of testosterone is unknown. However, it has been suggested that ginger may stimulate the production of luteinizing hormone (LH) which in turn more stimulate Leydig cells to produce testosterone (34). The effect of $Z$. officinal roots on fertility in diabetic male rats has been reported previously. It can enhance the serum testosterone and improve the sperm motility (35). In a study ginger which was added to semen fluid could induce toxic effects on sperm motility and morphology. The result was dose and time-dependent, but in several studies, ginger showed useful effects on the semen parameters (36).

\section{Conclusion}

The oral administration of $Z$. officinale hydro-alcoholic extract has been shown to increases body weight, reduce blood glucose concentration and improve serum insulin levels in STZ-induced diabetic rats. However, the extract significantly decreased HMG-COA reductase level in diabetic rat's testis. In general, ginger shows effective glycaemic control properties, and, has a potential influence in the regulation of cholesterol homeostasis through modulation of HMG-COA reductase level in testis of diabetic rats.

\section{Acknowledgements}

This research project was financially supported by hyperlipidemia research center at Ahvaz Jundishapur University of Medical Sciences, Ahvaz, Iran (Research Grant Number: HLRC-9501).

\section{Authors' contributions}

GM coordinated, designed, analyzed the data and revised the manuscript. AK provided assistance for study design and western blot analysis. SAP and NLD participated in plant extraction and all animal intervention. BMP and FA participated in the draft preparation and carried out western blot analysis. The paper has been read and approved by all authors.

\section{Conflict of interests}

The authors declared no conflict of interests exist.

\section{Ethical considerations}

The study was approved and conducted in accordance with the Ethical Committee of Animal Breeding and Research of Jundishapur University of Medical Sciences (Ethical Code: IR.AJUMS.REC.1395.25). thical issues have been observed by the authors.

\section{Funding/Support}

This research was financially supported by the Hyperlipidemia Research Center, Ahvaz Jundishapur University of Medical Sciences, Ahvaz, Iran (Research Grant Number: HLRC-9501).

\section{References}

1. Daniels TF, Killinger KM, Michal JJ, Wright RW Jr, Jiang Z. Lipoproteins, cholesterol homeostasis and cardiac health. Int J Biol Sci. 2009;5(5):474-88.

2. Williams KJ. Molecular processes that handle -- and mishandle -- dietary lipids. J Clin Invest. 2008;118(10):3247-59. doi: $10.1172 /$ jci35206.

3. Brown MS, Goldstein JL. A receptor-mediated pathway for cholesterol homeostasis. Science. 1986;232(4746):3447. doi: 10.1126/science.3513311.

4. Rudling M. Hepatic mRNA levels for the LDL receptor and HMG-CoA reductase show coordinate regulation in vivo. J Lipid Res. 1992;33(4):493-501.

5. Russell DW. Cholesterol biosynthesis and metabolism. Cardiovasc Drugs Ther. 1992;6(2):103-10. doi: 10.1007/ bf00054556.

6. Chen CW, Cheng HH. A rice bran oil diet increases LDL- 
receptor and HMG-CoA reductase mRNA expressions and insulin sensitivity in rats with streptozotocin/ nicotinamide-induced type 2 diabetes. J Nutr. 2006;136(6):1472-6. doi: 10.1093/jn/136.6.1472.

7. Han KH, Iijuka M, Shimada K, Sekikawa M, Kuramochi $\mathrm{K}$, Ohba K, et al. Adzuki resistant starch lowered serum cholesterol and hepatic 3-hydroxy-3-methylglutarylCoA mRNA levels and increased hepatic LDL-receptor and cholesterol 7alpha-hydroxylase mRNA levels in rats fed a cholesterol diet. Br J Nutr. 2005;94(6):902-8. doi: 10.1079/BJN20051598.

8. Shibata S, Hayakawa K, Egashira Y, Sanada $H$. Hypocholesterolemic mechanism of Chlorella: Chlorella and its indigestible fraction enhance hepatic cholesterol catabolism through up-regulation of cholesterol 7alpha-hydroxylase in rats. Biosci Biotechnol Biochem. 2007;71(4):916-25. doi: 10.1271/bbb.60566.

9. Li Y, Tran VH, Duke CC, Roufogalis BD. Preventive and Protective Properties of Zingiber officinale (Ginger) in Diabetes Mellitus, Diabetic Complications, and Associated Lipid and Other Metabolic Disorders: A Brief Review. Evid Based Complement Alternat Med. 2012;2012:516870. doi: 10.1155/2012/516870.

10. Rahmani AH, Shabrmi FM, Aly SM. Active ingredients of ginger as potential candidates in the prevention and treatment of diseases via modulation of biological activities. Int J Physiol Pathophysiol Pharmacol. 2014;6(2):125-36.

11. Abdulrazaq NB, Cho MM, Win NN, Zaman R, Rahman MT. Beneficial effects of ginger (Zingiber officinale) on carbohydrate metabolism in streptozotocin-induced diabetic rats. Br J Nutr. 2012;108(7):1194-201. doi: $10.1017 / \mathrm{s} 0007114511006635$.

12. Bhandari U, Sharma JN, Zafar R. The protective action of ethanolic ginger (Zingiber officinale) extract in cholesterol fed rabbits. J Ethnopharmacol. 1998;61(2):167-71. doi: 10.1016/S0378-8741(98)00026-9.

13. Verma SK, Singh M, Jain P, Bordia A. Protective effect of ginger, Zingiber officinale Rosc on experimental atherosclerosis in rabbits. Indian J Exp Biol. 2004;42(7):736-8.

14. Bhandari U, Kanojia R, Pillai KK. Effect of ethanolic extract of Zingiber officinale on dyslipidaemia in diabetic rats. J Ethnopharmacol. 2005;97(2):227-30. doi: 10.1016/j.jep.2004.11.011.

15. Fuhrman B, Rosenblat M, Hayek T, Coleman R, Aviram M. Ginger extract consumption reduces plasma cholesterol, inhibits LDL oxidation and attenuates development of atherosclerosis in atherosclerotic, apolipoprotein E-deficient mice. J Nutr. 2000;130(5):1124-31. doi: 10.1093/jn/130.5.1124.

16. Thomson M, Al-Qattan KK, Al-Sawan SM, Alnaqeeb MA, Khan I, Ali M. The use of ginger (Zingiber officinale Rosc.) as a potential anti-inflammatory and antithrombotic agent. Prostaglandins Leukot Essent Fatty Acids. 2002;67(6):475-8. doi: 10.1054/plef.2002.0441.

17. Ahmed RS, Sharma SB. Biochemical studies on combined effects of garlic (Allium sativum Linn) and ginger (Zingiber officinale Rosc) in albino rats. Indian J Exp Biol. 1997;35(8):841-3.
18. Srinivasan K, Sambaiah K. The effect of spices on cholesterol 7 alpha-hydroxylase activity and on serum and hepatic cholesterol levels in the rat. Int J Vitam Nutr Res. 1991;61(4):364-9.

19. Lamuchi-Deli N, Aberomand M, Babaahmadi-Rezaei H, Mohammadzadeh G. Effects of the Hydroalcoholic Extract of Zingiber officinale on Arginase I Activity and Expression in the Retina of Streptozotocin-Induced Diabetic Rats. Int J Endocrinol Metab. 2017;15(2):e42161. doi: 10.5812/ijem.42161.

20. Harris MI. Hypercholesterolemia in diabetes and glucose intolerance in the U.S. population. Diabetes Care. 1991;14(5):366-74. doi: 10.2337/diacare.14.5.366.

21. Kannel WB, McGee DL. Diabetes and glucose tolerance as risk factors for cardiovascular disease: the Framingham study. Diabetes Care. 1979;2(2):120-6. doi: 10.2337/ diacare.2.2.120.

22. Prevalence of small vessel and large vessel disease in diabetic patients from 14 centres. The World Health Organisation Multinational Study of Vascular Disease in Diabetics. Diabetes Drafting Group. Diabetologia. 1985;28 Suppl:615-40.

23. Saraswat M, Suryanarayana P, Reddy PY, Patil MA, Balakrishna N, Reddy GB. Antiglycating potential of Zingiber officinalis and delay of diabetic cataract in rats. Mol Vis. 2010;16:1525-37.

24. Kusari J, Zhou S, Padillo E, Clarke KG, Gil DW. Effect of memantine on neuroretinal function and retinal vascular changes of streptozotocin-induced diabetic rats. Invest Ophthalmol Vis Sci. 2007;48(11):5152-9. doi: 10.1167/ iovs.07-0427.

25. Eleazu CO, Iroaganachi M, Okafor PN, Ijeh, II, Eleazu KC. Ameliorative potentials of Ginger (Z. officinale Roscoe) on relative organ weights in streptozotocin induced diabetic rats. Int J Biomed Sci. 2013;9(2):82-90.

26. Faried MA, Mansour FK, Zolfakar AS, El-Kholy WB. Protective role of Ginger on the induced diabetic retinopathy in adult albino rats. J Am Sci. 2014;10(10):134-52.

27. Thomson M, Al-Amin ZM, Al-Qattan KK, Ali M. Hypoglycemic effects of ginger in mildly and severely diabetic rats. Faseb J. 2007;21(5):A50.

28. Zafar M, Naeem-Ul-Hassan Naqvi S. Effects of STZinduced diabetes on the relative weights of Kidney, liver and pancreas in albino rats: A comparative study. Int J Morphol. 2010;28(1):135-42. doi: 10.4067/S071795022010000100019.

29. Iranloye BO, Arikawe AP, Rotimi G, Sogbade AO. Antidiabetic and anti-oxidant effects of Zingiber officinale on alloxan-induced and insulin-resistant diabetic male rats. Niger J Physiol Sci. 2011;26(1):89-96.

30. Akhani SP, Vishwakarma SL, Goyal RK. Anti-diabetic activity of Zingiber officinale in streptozotocin-induced type I diabetic rats. J Pharm Pharmacol. 2004;56(1):1015. doi: 10.1211/0022357022403.

31. Jung UJ, Lee MK, Park YB, Kang MA, Choi MS. Effect of citrus flavonoids on lipid metabolism and glucoseregulating enzyme mRNA levels in type-2 diabetic mice. Int J Biochem Cell Biol. 2006;38(7):1134-45. doi: 10.1016/j.biocel.2005.12.002. 
32. Al-Amin ZM, Thomson M, Al-Qattan KK, PeltonenShalaby R, Ali M. Anti-diabetic and hypolipidaemic properties of ginger (Zingiber officinale) in streptozotocininduced diabetic rats. Br J Nutr. 2006;96(4):660-6. doi: 10.1079/BJN20061849.

33. Kamtchouing P, Mbongue Fandio GY, Dimo T, Jatsa HB. Evaluation of androgenic activity of Zingiber officinale and Pentadiplandra brazzeana in male rats. Asian J Androl. 2002;4(4):299-301.

34. Mares AK, Abid W, Najam WS. The effect of Ginger on semen parameters and serum FSH, LH \& testosterone of infertile men. Tikrit Med J. 2012;18(2):322-9.

35. Shalaby MA, Hamowieh AR. Safety and efficacy of Zingiber officinale roots on fertility of male diabetic rats. Food Chem Toxicol. 2010;48(10):2920-4. doi: 10.1016/j. fct.2010.07.028.

36. Akhlaghi A, Ahangari YJ, Navidshad B, Pirsaraei ZA, Zhandi $M$, Deldar $H$, et al. Improvements in semen quality, sperm fatty acids, and reproductive performance in aged Cobb 500 breeder roosters fed diets containing dried ginger rhizomes (Zingiber officinale). Poult Sci. 2014;93(5):1236-44. doi: 10.3382/ps.2013-03617. 